\title{
Human Consciousness: The Universal Heart Based Resonant Frequencies and the Massive ecosystems Hierarchy
}

\author{
Abdullah A Alabdulgader* \\ Congenital Cardiologist \& Invasive Electro physiologist, Prince Sultan Cardiac Center, Saudi Arabia
}

*Corresponding author: Abdullah Al Abdulgader, Congenital Cardiologist \& Invasive Electro physiologist, Prince Sultan Cardiac Center, P.O.Box 9596, Hofuf, Al-Ahsa 31982, Saudi Arabia.

Received Date: October 15, 2020

Published Date: November 02, 2020

\section{Mini Review}

Few scientific dilemmas in the beginning of the third decade of the 21st century, are still not investigated in an integral comprehensive approach compatible with the era scientific advances. Human Consciousness, its origin, nature and the delicate symphony orchestrating its execution remains at the top of the list of the elusive sciences of the human history. Intuitively, our ancestors refer to the human heart as the seat of soul and the center of the consciousness experience. The scientific evidence of this miraculous issue must be life time struggle but scientistists in quantum physics, cardiac astrophysics, mathematics and computational engineering in the last 8 decades started to decode the miraculous mystery. Five international conferences, King of Organs Conferences (KOO) chaired by the author of this review were held (King of Organs International Conferences for Advance Cardiac Sciences in 2006,2008, 2010,2012 and2019) with collaboration of collective group of world leaders in cardiac astrophysics, stress, heart math, chronobiology, mathematics, geology and space sciences. The great collective mission was to navigate in consciousness scientific aspects. KOO series of conferences started in 2006, incorporated up to date scientific understandings in the field of consciousness in the last 80 years, with unique and special scientific direction considering the human heart as central operator in the consciousness experience. Escalating accumulation of scientific evidences of what was thought of as intuitive concepts about the human heart role of consciousness had became evident, the dawn of which emerged in respectful publications from $\mathrm{KOO}$ and scientific collaborates research. There is compelling scientific and rational evidence to convince scientific communities that the nature of consciousness involves dynamics inside the skull but essentially much beyond it in extreme dimensions between the skull and the sky. The approach we adopted is based on holistic perspective and understanding of human consciousness incorporating quantum physics, cardiac astrobiology, mathematics, neurocardiology, and other disciplines in addition to philosophical bases of consciousness. Scrutinized investigation of the consciousness science and its implications and consequent regulations in medical practice illustrated clearly that major proportion of the misleading implementation of decisions to end life were related directly to the faulty and drastically deficient definition of consciousness. Recently consciousness research refers to the Consciousness Tetrad which describes four escalating levels of consciousness starting from the default consciousness which is the default state that separates the living from the dead, rising to the aware consciousness which looks at consciousness as a continuum of states ranging from awake to sleep to drowsiness to semiconscious states like stupor and finally coma. The third level is the operational consciousness which is consciousness related to motor, sensory, cognitive, ethical, creative, emotive, and other abilities and capabilities and awareness of all mental operations. The fourth level is what philosophers refers to as the transcendence 
of human soul called the exalted consciousness where the most elite and spiritual experience of a human being can be achieved as the person is getting closer to the thresholds of the highest intelligence ever, who masters the emergence and fate of death and life and all creatures and universes. The author defines Consciousness as a state of alertness and being aware, active and vigilant of the self and surroundings with volition, based in memory and personal identity. This state is ineffable and intrinsic and express itself in presence of soul through activation of different sensing and perceptive body organs but may pass through lighter densities and variable dimensions in quantum nature, if soul leaves the body. The level of human consciousness is the collective activity of widespread areas of bilateral association of cortical and subcortical structures and other interconnected biological and astrophysical systems. Although much has been learned about the neuroanatomical structures participating in consciousness, there is always great demand to establish the physiological and physical mechanisms through which consciousness is generated in these networks, structures and dimensions. In the last 7 decades the neuroscientific communities were somewhat limited and trapped in the limitations of the neuronal firing theory of the reductionist. The father of the reductionist theory of brain functioning in todays medicine is Wilder Penfield's (1891-1976) who adopt the concept that electrical stimulations in certain brain areas produce experiential phenomena [1]. Th dogma of almost all medical communities now a days are relating consciousness to "brain related consciousness systems" including cortical components, subcortical components comprising multiple parallel arousal systems in the upper brainstem, thalamus, hypothalamus, and basal forebrain, cerebellum as well as sensory and motor components and neurotransmitter pathways. It is astonishing for us at this stage of history to review the scientific jewels of the consciousness quantum theories of the heroes of quantum physics starting from the early twentieth century until now which has been neglected in the creation of scientific perspective of the realm of consciousness. In historical appraisal R. Nitsch and F. W. Stahnisch in the journal Cerebral Cortex challenged Penfield original concept of experiential phenomena elicited by electrical Stimulation of the human cortex. They revisited Penfield clinical work and found that the actual results obtained from electrical stimulation studies of the brain are far less conclusive, than his firm assertions made during Penfield Gordon Wilson Lecture in 1950 [2]. It is astonishing to all medical communities to know that The thalamocortical complex does not seem to be critically essential for consciousness experience. Brainstem mechanisms by its own can create adequate consciousness state. The prove came up from real life experience with series of publications on Hydranenecephalic children. Neurological evaluation reveals they are responsive to their surroundings and conscious. Personal observations reported by hundreds of families of affected children stressed on the fact that their responsiveness is most readily to sounds, but also to salient visual stimuli [3]. The neuroanatomical components of the human brain and the brain mass seems to bear no true correlation to intelligence. The brain of the most intelligent human in the 20 century,claimed to be, Albert Einstein was not showing any convincing anatomical difference than any body brain [4]. Lionel Feuillet et al, published in the Lancet 44 years old French man with otherwise normal neurological development and medical history who was found with $90 \%$ absence of his cerebral cortex. His consciousness, mentality as well as social life were otherwise normal [5]. Orchestration of the human Consciousness from beyond the brain became increasingly accepted scientific discussion. The last 25 years witnessed revolutionary shift in the deep understanding of the role of non neurobiological aspects, beyond the skull in the consciousness experience in humans. Advances especially, our work and others, in the science of Heart Rate Variability[HRV] and the role of ascending neuronal afferents from the heart to the brain in addition to the miraculous universal symphony between human heart and the universe constitute the most important revolutions in the new understanding [6-11]. Adopting the quantum consciousness theories in the new understanding based on human heart energetic interaction with multiple energetic fields and frequencies constitute the bases of our new understanding of consciousness experience. Advances in neurocardiology and the heart brain neurodynamics contribute significantly to the new perspective for cardiac dominance in certain physical aspects affecting consciousness. In specific heart electromagnetic field was found to have striking dominance over brain magnetic field. The amplitude of the cardiac electrical signal was found to be 60 times greater compared to the brain signal while the electromagnetic field of the heart is approximately 5000 times stronger than the brain and can be detected six feet away from the body with sensitive magnetometers. John and Beatrice Lacey during 1960s and 1970s created a massive drift in the modern psychophysiological research with their publications on human heart -brain communication [12]. An important land mark in the field was there observations that afferent input from the heart and cardiovascular system could significantly affect perception,cognitive functions and behavior. Cognitive performance was found to be fluctuated around a rhythm frequency of $0.1 \mathrm{~Hz}$. This was demonstrated by Velden, Wolk. Lane RD, Thayer JF, and collaborates who showed that the modulation of cortical function was via the heart's influence due to afferent inputs on the neurons in the thalamus, which globally synchronizes cortical activity and the consciousness phenomena $[13,14]$. A critical observation in this regard is the finding that "pattern and stability" [of the rhythm] of the heart's afferent inputs, rather than the number of neural bursts within the cardiac cycle that will modulate thalamic activity, which 
in turn has global effects on brain function and ultimately the consciousness experience. [15]. Revolutionary paradigm, is prevailing with the documentation that the heart detect stimulus before the brain and brain neural events are locked to heartbeats [16]. Rigorous experimental study demonstrated the heart receives and processes information about a future event before the event actually happens. Rollin McCraty study's results provide surprising data showing that both the heart and brain receive and respond to pre-stimulus informaᄀtion about a future event before it occurs but the heart proceeded the brain by 1.3 seconds which is truly too long time in the scale of neural impulse transmission that is counted with milliseconds [17]. We and our team are contributing to new understanding implying that VAGUS NERVE IS NEVER VAGUS. In contrary, $85-90 \%$ of the fibers in the vagus nerve are afferent communicating the state of the body to the brain [18]. The afferent vagal pathways consists of the nerve structures that carry information from the body to the brain stem and cerebral cortex [19]. The majority of higher brain centers, including the thalamus, hypothalamus, and amygdala as well as emotional experience and cognitive processes are operated by Cardiovascular related afferent neural traffic [20]. Afferent input from the heart affects the homeostatic regulatory centers in the brain, as well as the activity of higher brain centers involved in perceptual, cognitive, and emotional processing, thus in turn affecting many and diverse aspects of our experience, behavior and conscious experience [17]. Range of frequencies of complex afferent information related to mechanical and chemical factors is continuously sent to the brain and is over time scales ranging from milliseconds to minutes [21]. Clinical works in different disease states prove the neuroanatomical connections and physiological effects of vagus nerve higher innervations. Activating afferent input with vagal nerve stimulation[VNS] is apparently heralding a new era in medical therapeutics as it proves effective in many psychophysiological disorders including epilepsy, obesity, depression, anxiety, autism, alcohol addiction, mood disorders, as well as multiple sclerosis, and traumatic brain injury [22,23]. The vagal afferent connections have multiple consciousness related targets, which include the thalamus, amygdala, reticular formation, hippocampus, raphe nucleus, and the locus coeruleus. VNS will create improved global neurostimulation state leading to promoted spread of cortical signals and caused an increase of metabolic activity leading to behavioral improvement as measured with the Coma Recovery Scale-Revised[CRS-R] scale [23,24]. Vagus nerve stimulation[VNS] may also contribute to breaking advances in awakening the unconscious vegetative state patient as approved by improvement in behavioral responsiveness and enhanced brain connectivity patterns [25]. Since the vagal efferents dominate cardiac control [26], and the vagus contains a high percentage of afferent fibers [27], it is theorized that both the input and output of the Central
Autonomic Network [CAN] in the brain are directly linked to HRV [28]. The science of Heart Rate Variability[HRV] and its accurate reflection of Autonomic Nervous System[ANS] functions created striking revolution in our understanding of the body psychophysiological well being as well as the human heart near and far communications with other humans and the ecosystems around us. It is astonishing to know that chaotic HRV wave form is associated with disturbed emotions and described as cortical inhibitor, while sine wave smooth appearance of the HRV is associated with cortical facilitation and higher intelligence. This last situation where described by McCraty and colleagues as a state of psychophysiological coherence to describe the degree of order, harmony, and stability in the various rhythmic activities within living systems over any given time period $[15,29]$. This cardiac coherence can be measured by HRV analysis where more ordered sine like HRV pattern will be seen spiking around frequency of 0.1 $\mathrm{Hz}$ [10 seconds] represented by very narrow, high-amplitude peak in the low frequency [LF] region of the HRV power spectrum with no major peaks in the VLF or HF regions [30]. Ground breaking discovery emphasizing the ability of afferent cardiac signals to reprogram the cortical and subcortical neural networks is what we describe as the repatterning process in the neural architecture, where coherence becomes established as a new, stable baseline reference memory with resultant psychophysiological well being [31]. The establishment of psychophysiological coherence is the role of the heart with its cortical and subcortical reprogramming capabilities, to create a state of escalating consciousness from default, awareness and operational consciousness to exalted consciousness with true transcendence experience. An amazing discovery in the field of cardiac neural input to the brain is the Heat Beat Evoked Potentials [HBEPs] representing the heart's afferent neurological input to the brain which are detectable between 50 and $550 \mathrm{~ms}$ after each $\mathrm{R}$ wave in the EEG. The author used to call it cardiac consciousness signature in the brain. Hyeong-Dong Park et al, found that neural responses to heartbeats can be recorded mainly in the insula [i.e., anterior, posterior] which is the primary cortical projection site of interoceptive signals reflecting self consciousness experience. HBEPs can be seen also in operculum [i.e., frontal, central, posterior] the amygdala and fronto-temporal cortex [32]. An other striking development which creates historical turning point in the consciousness sciences time line is the discovery of the extreme delicate orchestration between human heart interbeats variability [HRV]- here to close the statement started with its impact on psychophysiological and consciousness experience, and the planetary and galactic energies. Pioneering efforts in this field were established by Franz Halberg, Rollin McCraty, Richard Gevirtz and others [6-11,17,20,25,30,31,32,34] Our team was able to achieve the longest record in human history of HRV of 16 individuals for 96000 hours which was managed 
statistically using multivariate linear regression analysis with Bonferroni corrections for multiple comparisons. The HRV record were synchronized to Schumann Resonances [SR], Solar Wind Indices and Galactic Cosmic Rays [GCR] [33]. Increase in GCR, solar radio flux, and Schumann Resonance power was all associated with increased HRV and parasympathetic activity [HF power]. For solar radio flux [F10.7] which is the integrated solar flux between 2750 and $2850 \mathrm{MHz}$ we found an immediate, robust positively correlated response with four HRV important parameters: the inter beat interval [ IBIs],total power[TP], the very low frequency band [VLF] and the high frequency band[ $\mathrm{HF}]$ power. There is an inverse relationship between the F10.7 and death rates from various causes [34]. The positive correlation found between HF power and solar radio flux indicates an enhancement of parasympathetic nervous system activity during periodsof increased solar radio flux. Vagally [parasympathetic] mediated HRV has been linked to raised consciousness state and optimal psychophysiological well being in normal people and to drastically improve vegetative state to minimal consciousness $[7,15,17,23]$. The resonance between human heart frequencies with the universal energetic levels of this long period is a breaking news in the field which we integrate very well with quantum consciousness theories in one global perspective to understand the mystery of human consciousness. It is conceivable now that neuronal firing of brain structures is convincingly not enough to explain subjective consciousness experience. The idea that quantum mechanics has something to do with the workings of the mind was developed by Eugene Wigner, Hungarian-American theoretical physicist and Nobel Prize Laurete in Physics in 1963, who proposed that the wave function collapses due to its interaction with consciousness. In recent scientific breakthrough the Global Consciousness Project [GCP] lead by Roger Nelson and Helané Wahbeh provided recent evidence supporting the hypothesis that our consciousness can even influences our physical world. They adopt Random number generators [RNGs] as a tool used to evaluate micro-psychokinesis or our ability to affect the physical world with our consciousness. Roger Nelson who is the founder of GCP reported in a recent publication multiple examples of striking similarity between event-related brain potentials and event-related correlations in random data [35]. Quantum physicists Larissa Brizhik and Emilio DelGiudice suggested that the most likely physical agent that can continuously provide an exchange of information between living systems within the larger ecosystem is the magnetic fields. The quantum field theory, denotes that potentials of the magnetic field, governs the dynamics of biological systems and the whole ecosystem. Much of our quantum understanding is influenced by David Bohm's views who perceives mind and matter as projections into our explicate order from the underlying implicate order. An energy-based concept of information was described by Dennis Gabor, who invented the hologram in 1947, which he described as quanta of information. Kal H. Pribram's holonomic model of brain processing was described in his 1991 Brain and Perception book which include his perspectives on human consciousness with David Bohm. It describes human cognition by modeling the brain as a holographic storage network. Pribram suggests these processes involve electric oscillations in the brain's dendritic networks, which are different from the more commonly known action potentials involving axons and synapses. Pribram's holonomic model contributes significantly to human consciousness understanding, specially to the fast associative memory and the non-locality of memory. In 1991 Roger Penrose who is Nobel laureate for physics and Stuart Hameroff who is anaesthesiologist and psychologist introduced the Orchestrated Objective Reduction [Orch-OR] theory which is a biological philosophy of mind which postulates that at the quantum level consciousness originates inside neurons, rather than the traditional perspective that it is a product of connections between neurons. Their mechanism is based on non computational quantum process performed by quantum bits [qubits] formed collectively on cellular microtubules- called objective reduction. In 1967 Hiroomi Umezawa and collaborators proposed a quantum field theory of memory storage which is fundamentally different from the PenroseHameroff theory based on quantum theory of the brain which posits a spatially distributed charge formation exhibiting spontaneous breakdowns at micro levels as the basis for processing at macro levels. According to this model, the information resides in the virtual field associated with the dynamics of the cellular matter. The approach of Hiroomi Umezawa was built upon by Karl Pribram and many others and expanded by Giuseppe Vitiello to a dissipative quantum model of brain. Henry Pierce Stapp an other pioneer in quantum consciousness arena, postulates a process of global collapse through an effect on the synapses by exploitation certain aspects of quantum Zeno effect. Each theory discussed has its strength and weaknesses but all lack the comprehensive universal perspective incorporating the origin of consciousness with more homogenous incorporation of current theories in a stronger model capable of bringing us closer to consciousness realm closer than any time ever. The most comprehensive explanation of consciousness events in the medical literature up to date was the description of Stuart Hameroff, RogerPenrose in their review in 2014 when they stated that Biology evolved a mechanism to orchestrate such events and to couple them to neuronal activity,resulting in meaningful, cognitive, conscious moments and thence also to causal control of behavior [36]. Again it lacks any indication of the source of the orchestrated consciousness, its ecosystemic hierarchy and the mechanism where the macro cosmic systems interact with the microbiology of our living cells. The focus of the previous theories on quantum consciousness was more of micro perspective with little of the macro holistic perspective. In continuation to the brilliant mentioned theories we are postulating science based model of the evolutionary dynamics of consciousness 
experience as a series of universal events. Our point of view represents our own perspectives after 14 years of research and scientific debate in 5 international conferences that we founded and chaired "King of Organs International Congress for Advanced Cardiac Sciencec,2006, 2008, 2010, 2012, and 2019" in collaboration with Heart Math Istitute [Boulder Creek, California, USA], the American Institute of Stress[AIS][NY,USA] the Global Consciousness Project[GCP] [USA] and others. The human heart has been conceived of throughout time and across Western and Eastern traditions as a holy organ and the hub of perception and sacred awareness. In ancient medical classics from Babylon, China, Egypt, Greece, Rome and Islamic civilizations, it is described as the center of a system supplying life force to the body and the seat of consciousness [37,38]. This intuitive perception of the role of human heart as orchestrator of the human consciousness experience which received honor and sacricm a long the human history, was not supported by compelling scientific evidence. Our theory [Alabdulgader Theory of Consciousnesss, The Heart Based Resonant Fields [HBRF]] is based on human heart as the center of human consciousness experience that orchestrate with the planetary and cosmic energetic fields. We were able to achieve the longest record of human Heart Rate Variability [HRV][96000 hours] synchronized with the near planetary frequencies, namely the Shumann resonances[SR] and the far, namely Solar Wind indices and the extremely far Cosmic frequencies originating from the endless universal nuclear explosions, namely the Galactic Cosmic Rays[GCR] [33]. Complementary to our hypothesis is the heart magnetic field interactions with the brain neuronal electrical fields as described by Karl Pribram as well as the quantum bit state reduction on micro tubules proposed by Penrose and Hamerof and Hiroomi Umezawa and collaborators who proposed a quantum field theory of memory storage. Our hypothesis is based on postulating that there is gigantic source of information contains the most delicate details of the emergence and the fate of all life events and all galaxies and creatures within it. The information is documented and preserved with infinitive accuracy in the form of endless gigantic nuclear energetic fields that we call the Preserved Board[PB].The eternal and the eterral governer of the PB is the miraculous intelligent consciousness of the whole polyverse and all life and beyond. In our domain we call him ALLAH. Our hypothesis of the global consciousness including human consciousness is based on three levels of energetic fields. First is the source of the energy, namely the $\mathrm{PB}$, second is the media of information transmission, namely the massive electrosoliton and the potential magnetic patterns and fields transmitted through the galaxies until earth, third is the receiver,the discriminator, and the magnetic patterns generator namely the the heart and its pulsating magnetic field and associates in addition to the entangled electromagnetic fields, neurobiological processes of the brain and electrodermal response. Electrosolitons, which are the charge, energy, and information carriers in the metabolic processes in biological systems, according to Davydov's hypothesis [39], are formed in alpha-helical proteins, DNA, and hydrogen-bonded water molecule chains, present in living cells, intercellular tissues, and in all ecological systems. The electromagnetic fields of the universal systems and its associated potential patterns is the information carrier. The dynamic mass of the electrosoliton depends on the frequency of the magnetic field which affect charge transport processes. At the resonant frequency, electrosoliton wave will be drifted by the magnetic field and accordingly emit radiation and sound waves intensively then lose their energy. Electrosolitons emit their own electromagnetic radiation with the frequency of the main harmonic which is dependent on their own velocity. Electromagnetic potential can cause the emergence of the coherent structures that in view of their coherence, openness, and nonlinearity, are able to self-organize and form various hierarchical levels of ecosystems [40]. Via this electrosoliton emitted electromagnetic radiation, subunits of the complex system [cells, organs, individuals, ecosystems] exchange their information which leads to the appearance of the long-range order in a process of the massive hierarchy of ecosystems that is on going in descending manner from the galaxies to the local electromagnetic field [EMF] of the heart and brain with impressive accuracy. We have scientific evidence that our hearts represented by HRV activity -which is an accurate indicator of the human psychophysiological and consciousness state - are in a state of universal symphony with the near and far cosmic environments [15,33] There is a direct mathematical relationship between the HRV patterns which contains our magnetic potentials of our emotions and the spectral information encoded in the magnetic field [41]. This ultimately means that our emotions and consciousness are truly pulsating and resonate with the far universe in highly organized hierarchical manner. Our new perspective of human consciousness can blend easily in complementary manner with David Bohm's model of the implicate order and its projection, or extrusion into space-time, and with Karl Pribram holonomic mind/brain theory. The Higgs boson and the Higgs field theory is in complete accordance with our hypothesis. When the descendant information from the PB transformed by the magnetic fields- emitted from the interaction of magnetic fields with the massive electrosoliton wave- it interacts with the human heart EMF and brain neuronal entangled fields and neuronal synapses. Psychokinetic process will take over where several encoded choices within the descendant magnetic potential patterns will interact with heart EMF and brain neuronal entangled fields. According to the wave function collapse, the descendant magnetic potential patterns will be reduces to a single pattern at variable time intervals. Von Neumann-Wigner interpretation mentioned about "consciousness causes collapse" in the context of quantum mechanics as general description of the consciousness experience. Psychokinetic affect of all humanity on collective global 
consciousness can be thought of with the size of energetic levels fed by over 7 billion beating human hearts to the space. This would be the source of a radiant magnetic potentials energy feeding into an evolving planetary fields. Our postulated underlying mechanism for this conscious decision making step of the HBRF theory is a dual effect of an interaction of the descendant magnetic potential and the neuronal entanglement fields as described by Karl Pribram and the global process collapse through an effect on the synapses with means of quantum Zeno effect as described by Henry Pierce Stapp . Freeman Dyson argument that mind as manifested by the capacity to make choices, is inherent in every electron is in a strong concordance to the wave collapse part of our HBRF theory. We postulate this wave function collapse is the last step occurs between the cerebral neuronal entanglement fields and neuronal synapses interacting with the descendant magnetic potentials before final decision is taken. The heart afferent neuronal inputs to cortical and sub cortical systems and the associate neurotransmitter pathways, are a major player in this process of decision making of one choice over the other. This higher level of orchestrated consciousness from the PB to the neurobiology of heart and brain is confined exclusively to human kind for which they are committed to the higher power being responsible about their choices in life.

\section{Acknowledgement}

None.

\section{Conflict of Interest}

No conflict of interest.

\section{References}

1. Penfield W (1938) The cerebral cortex in man: I. The cerebral cortex and consciousness. Arch Neurol Psychiatry 40(3): 417-442.

2. R Nitsch, FW Stahnisch (2018) Neuronal Mechanisms Recording the Stream of Consciousness-A Reappraisal of Wilder Penfield's (18911976) Concept of Experiential Phenomena Elicited by Electrical Stimulation of the Human Cortex. Cerebral Cortex 28(9): 3347-3355.

3. Bjorn Merker (2007) Consciousness without a cerebral cortex: A challenge for neuroscience and medicine. Behavioral and Brain Sciences 30: 63-81.

4. M Galaburda (1999) Albert Einstein's brain. THE LANCET.

5. Lionel Feuillet, Henry Dufour, Jean Pelletier (2007) Brain of a whitecollar worker. Lancet 370: 262.

6. Timofejeva I, McCraty R, Atkinson M, Joffe R, Vainoras A, et al. (2017) Identification of a Group's Physiological Synchronization with Earth's Magnetic Field. Int J Environ Res Public Health 14(9): 998.

7. McCraty R, Atkinson M, Stolc V, Alabdulgader AA, Vainoras A, et al (2017) Synchronization of Human Autonomic Nervous System Rhythms with Geomagnetic Activity in Human Subjects. Int J Environ Res Public Health 14(7): 770.

8. Abdullah Alabdulgade, Rollin McCraty, Mike Atkinson, Alfonsas Vainoras, Kristina Berškiene, et al. (2015) Human Heart Rhythm Sensitivity to Earth Local Magnetic Field Fluctuations. Journal of Vibroengineering17: 3271-3278.

9. Franz Halberg, Germaine Cornélissen, Rollin McCraty, Jerzy Czaplicki, Abdullah A Al Abdulgader (2011) Time Structures (Chronomes) of the Blood Circulation, Populations' Health, Human Affairs and Space Weather. World Heart Journal 3.
10. Abdullah A Al Abdulgader, Germaine Cornélissen Guillaume, Franz Halberg (2011) Vascular Variability Disorders in the Middle East: Case Reports. World Heart Journal.

11. Inga Timofejeva, Rollin McCraty, Mike Atkinson, Roza Joffe, Abdullah Alabdulgader, et al. (2018) Global Study of Human Heart Rhythm Synchronization with the Earth's Time Varying Magnetic Field. Underr review in the BMC Bioinformatics journal.

12. Lacey JI (1959) Psychophysiological approaches to the evaluation of psychotherapeutic process and outcome. In: Rubinstein E, Parloff M, (eds.), Research in Psychotherapy Washington, DC: American Psychological Association:160-208.

13. Wolk C, Velden M (1989) Revision of the baroreceptor hypothesis on the basis of the new cardiac cycle effect. In Psychobiology: issues and applications. Amsterdam: Elsevier Science Publishers B.V. 371-379.

14. Lane RD, Reiman EM, Ahem GL, Thayer JF (2001) Activity in medial prefrontal cortex correlates with vagal component of heart rate variability during emotion. Brain Cognit 47: 97-100.

15. Abdullah A Alabdulgader (2017) The human heart rate variability; Neurobiology of psychophysiological well being and planetary resonance. Editorial. Gen Int Med Clin Innov 2(2): 2-4

16. Hyeong-Dong Park, Stéphanie Correia, Antoine Ducorps, Catherine Tallon-Baudry (2014) Spontaneous fluctuations in neural responses to heartbeats predict visual detection. Nature Neuroscience 17: 612-618.

17. Rollin McCraty, Mike Atkinson, Raymond Trevor Bradley (2004) Electrophysiological evidence of intuition: Part 2. A system-wide process? Journal of Alternative and Complementary Medicine 10(2): 325-336.

18. Cameron OG (2002) Visceral Sensory Neuroscience:Interception. New York: Oxford University Press, USA.

19. Tortora GJ, Grabowski SR (1996) Principles of anatomy and physiology (8th ed.). New York: Harper Collins, USA.

20. McCraty R, Atkinson M, Tomasino D, Bradley RT (2009b) The coherent heart: heart-brain interactions, psychophysiological coherence, and the emergence of system wide order. Integral Rev 5: 10-115.

21. Armour JA, Kember GC (2004) Cardiacsensoryneurons in Basicand Clinical Neurocardiology. In: JA Armourand, JL Ardell (eds.), NewYork: Oxford University Press, USA, pp. 79-117.

22. Kosel M, Schlaepfer TE (2003) Beyond the treatment of epilepsy: new applications of vagus nerve stimulation in psychiatry. CNS Spectr 8: 515521.

23. Groves DA, Brown VJ (2005) Vagal nerve stimulation: are view of its applications and potential mechanisms that mediate its clinical effects. Neurosci Biobehav Rev 29: 493-500.

24. Tiller WA, McCraty R, Atkinson M (1996) Cardiac coherence: a new, noninvasive measure of autonomic nervous system order. Altern Ther Health Med 2: 52-65.

25. Corazzol M, Lio G, Lefevre A, Gianluca Deiana, Laurence Tell, et al. (2017) Restoring consciousness with vagus nerve stimulation. Curr Biol 27(18): R994-R996.

26. Levy MN (1990) Autonomic interactions in cardiac control. Annals of the New York Academy of Sciences 601: 209-221.

27. Porges SW (1995) Cardiac vagal tone: A physiological index of stress. Neuroscience and Biobehavioral Reviews 19: 225-233.

28. Thayer JF, Lane RD (2009) Claude Bernard and the heart brain connection: Further elaboration of a model of neurovisceral integration. Neuroscience and Behavioral Reviews 33(2): 81-88.

29. Rollin McCraty, Fred Shaffer (2015) Heart Rate Variability: New Perspectives on Physiological Mechanisms, Assessment of Selfregulatory Capacity, and Health Risk. Glob Adv Health Med 4: 46-61.

30. Bradley RT, McCraty R, Atkinson M, Tomasino D (2010) Emotion SelfRegulation, Psychophysiological Coherence, and Test Anxiety: Results 
from an Experiment Using Electrophysiologi-cal Measures. Applied Psychophysiology and Biofeedback 35(4): 261-283.

31. McCraty R, Atkinson Mike, Dana Tomasino, Raymond Trevor Bradley's (2006) The coherent heart: Heart-brain interactions, psychophysiological coherence, and the emergence of system-wide order. Boulder Creek, CA: Heart Math Research Center, Institute of Heart Math: 06- 022.

32. Hyeong Dong Park, Fosco Bernasconi, Roy Salomon, Catherine Tallon, et al. (2018) Neural Sources and Underlying Mechanisms of Neural Responses to Heartbeats, and their Role in Bodily Self-consciousness: An Intracranial EEG Study. Cerebral Cortex 28: 2351-2364.

33. Alabdulgader A, McCraty R, Atkinson M, Dobyns Y, Vainoras A, et al (2018) Long-Term Study of Heart Rate Variability Responses to Changes in the Solar and Geomagnetic Environment [Internet]. Sci Rep 8: 2663.

34. Stoupel, E. et al. (2011) Twenty years study of solar, geomagnetic, cosmic ray activity links with monthly deaths number (n-850304). Journal of Biomedical Science and Engineering 4: 426.

35. Roger D Nelson (2020) The Global Consciousness Project's EventRelated Responses Look Like Brain EEG Event-Related Potentials. Journal of Scientific Exploration 34(2): 246-267.
36. Stuart Hameroff, RogerPenrose (2014) Review Consciousness in the universe A review of the' OrchOR' theory. Physics of Life Reviews 11: $39-78$

37. Micheline R (2020) Anderson The Spiritual Heart. Religions 11: 506.

38. Abdullah Alabdulgader (2020) The Ancient Wisdom at Intersection with Modern Cardiac Sciences. Special issue. submitterd to Heart and Mind Journal (under review).

39. Davydov AS (1985) Solitons in Molecular Systems (2nd edn), Reidel: Dordrecht.

40. Brizhik L, Del Giudice E, Jorgensen,Marchettini N, Tiezzi E (2009) The role of electromagnetic potentials in the evolutionary dynamics of ecosystems. Ecol Modell 220(16): 1865-1869.

41. McCraty R, Atkinson M, Tomasino D, Bradley R (2009) The coherent heart: heartbrain interactions, psychophysiological coherence, and the emergence of system-wide order. Integr Rev 5(2): 10-115. 